\title{
Interaction of Genetic and Environmental Factors in a Drosophila Parkinsonism Model
}

\author{
Anathbandhu Chaudhuri, Kevin Bowling, Christopher Funderburk, Hakeem Lawal, Arati Inamdar, Zhe Wang, and \\ Janis M. O'Donnell \\ Department of Biological Sciences, University of Alabama, Tuscaloosa, Alabama 35487-0344
}

Catastrophic loss of dopaminergic neurons is a hallmark of Parkinson's disease. Despite the recent identification of genes associated with familial parkinsonism, the etiology of most Parkinson's disease cases is not understood. Environmental toxins, such as the herbicide paraquat, appear to be risk factors, and it has been proposed that susceptibility is influenced by genetic background. The genetic model organism Drosophila is an advantageous system for the identification of genetic susceptibility factors. Genes that affect dopamine homeostasis are candidate susceptibility factors, because dopamine itself has been implicated in neuron damage. We find that paraquat can replicate a broad spectrum of parkinsonian behavioral symptoms in Drosophila that are associated with loss of specific subsets of dopaminergic neurons. In parallel with epidemiological studies that show an increased incidence of Parkinson's disease in males, male Drosophila exhibit paraquat symptoms earlier than females. We then tested the hypothesis that variation in dopamine-regulating genes, including those that regulate tetrahydrobiopterin, a requisite cofactor in dopamine synthesis, can alter susceptibility to paraquatinduced oxidative damage. Drosophila mutant strains that have increased or decreased dopamine and tetrahydrobiopterin production exhibit variation in susceptibility to paraquat. Surprisingly, protection against the neurotoxicity of paraquat is conferred by mutations that elevate dopamine pathway function, whereas mutations that diminish dopamine pools increase susceptibility. We also find that loss-of-function mutations in a negative regulator of dopamine production, Catecholamines-up, delay the onset of neurological symptoms, dopaminergic neuron death, and morbidity during paraquat exposure but confer sensitivity to hydrogen peroxide.

Key words: Parkinson's disease; paraquat; dopamine regulation; neurodegeneration; dopaminergic neurons; environment

\section{Introduction}

Despite rapid progress recently in the identification of genes associated with familial Parkinson's disease (PD), the cause of $>90 \%$ of PD cases is unknown. The neuronal effects of chemical agents, most notably MPTP (1-methyl-4-phenyl-1,2,3,6tetrahydropyridine) (Langston et al., 1983) and pesticides such as rotenone and paraquat $\left(1,1^{\prime}\right.$-dimethyl- $4,4^{\prime}$-bipyridinium), have implicated environmental toxins in the induction of sporadic PD (Betarbet et al., 2000; McCormack et al., 2002; Uversky, 2004). However, the identification of causative agents and events and the identification of individuals at risk remain major challenges in understanding and controlling this neurodegenerative disease.

\footnotetext{
Received June 1, 2006; revised Jan. 2, 2007; accepted Jan. 21, 2007.

This work was supported by grants from the National Institutes of Health (GM62879) and the National Aeronautics and Space Administration (NAG2-1499) to J.M.0. We gratefully acknowledge fruitful discussions with members of the $0^{\prime}$ Donnell and Guy and Kim Caldwell laboratories. The Drosophila Stock Center (Bloomington, IN) and Jay Hirsh (University of Virginia, Charlottesville, VA) provided critical transgenic fly strains. Anti-Drosophila tyrosine hydroxylase antiserum was generously provided by Wendi Neckameyer (St. Louis University School of Medicine, St. Louis, MO). All HPLC analyses were expertly performed by research assistant Janna Brown.

Correspondence should be addressed to Dr. Janis M. O’Donnell, Box 870344, Department of Biological Sciences, University of Alabama, Tuscaloosa, AL 35487-0344. E-mail: jodonnel@bama.ua.edu.

A. Chaudhuri's present address: Department of Pharmacology and Experimental Neuroscience, University of Nebraska Medical Center, Omaha, NE 68198-5800.

C. Funderburk's present address: Emory University School of Medicine, Center for Behavioral Neuroscience, Atlanta, GA 30329.

Z. Wang's present address: Stower Institute for Medical Research, 1000 East 50th Street, Kansas City, M0 64110. D0I:10.1523/JNEUROSCI.4239-06.2007

Copyright $\odot 2007$ Society for Neuroscience $\quad$ 0270-6474/07/272457-11\$15.00/0
}

It is likely that genes expressed in dopaminergic neurons, in addition to those associated with familial parkinsonism, have roles in determining susceptibility to environmental toxicants. Genes that regulate dopamine (DA) homeostasis are potential candidates. Dysregulation of DA, in particular its release into the cytoplasm of dopaminergic neurons, contributes to oxidative cascades that are the immediate cause of the demise of neurons (Stokes et al., 1999). Moreover, the elevation of DA synthesis in response to a variety of stressors (Kumer and Vrana, 1996; De Bellis et al., 1999) may place dopaminergic neurons at greater risk for oxidative damage (Kim et al., 2005). However, association studies of polymorphisms within these genes have not yielded clear-cut candidates (Tan et al., 2000; Warner and Schapira, 2003). Use of genetically tractable organisms to model geneenvironment interactions has become an efficient means of identifying genetic risk factors (Bilen and Bonini, 2005; Cooper et al., 2006).

We tested the hypothesis that genetic variation in DA regulation is a PD susceptibility factor in Drosophila, using ingestion of paraquat to induce oxidative stress in strains bearing mutations in three genes. The gene pale ( ple) encodes tyrosine hydroxylase (TH) (Neckameyer and White, 1993), which catalyzes the first and rate-limiting step in the DA biosynthesis pathway. Punch $(\mathrm{Pu})$ encodes GTP cyclohydrolase I (GTPCH) (Mackay and O'Donnell, 1983), the rate-limiting enzyme in the synthesis of the TH-regulating cofactor tetrahydrobiopterin $\left(\mathrm{BH}_{4}\right)$. The third 
gene, Catecholamines-up (Catsup), is a negative regulator of DA production in Drosophila, acting post-translationally on GTPCH and TH (Stathakis et al., 1999). The Catsup protein is predicted to possess either six or seven transmembrane domains (Carbone et al., 2006) and to be a member of a large family that includes mammalian orthologs. Mutations in these genes are homozygous lethal, whereas heterozygotes are fully viable, fertile, and, with few exceptions, phenotypically normal. Therefore, these mutants are likely to model human populations in which cryptic variation in these genes is likely to exist.

Here we show that the herbicide paraquat induces parkinsonian symptoms in these Drosophila strains. We also demonstrate that Catsup mutant strains with hyperactivated DA synthesis, which might be expected to place the organism at increased risk for oxidative stress, are instead better able to cope with the effects of paraquat exposure. In contrast, compromised DA synthesis enhances susceptibility to paraquat-induced oxidative stress.

\section{Materials and Methods}

Drosophila strains and culture maintenance. Two strains were used as controls, both wild type for the DA-regulating genes under examination in this study, an isogenized Canton S line and a white-eyed mutant, $D f(1) w, y$. The latter was routinely used for HPLC assays of adult head extracts to genetically remove pteridine eye pigments that generate a high background and interfere with detection of brain responses. Comparison of the two controls established that they were virtually identical in oxidative stress sensitivity, including time of tremor onset and survival rates. $D f(1) w, y$ was crossed into the following mutant backgrounds: Catsup ${ }^{26}$ / $\mathrm{CyO}, \mathrm{Catsup}^{12} / \mathrm{CyO}, \mathrm{dp}$ cn $\mathrm{Pu}^{\mathrm{Z22}}$ a px sp/SM1, and $p l e^{2} / T M 3$. All stocks were maintained at $25^{\circ} \mathrm{C}$, under equivalent culture densities. All studies were conducted on mutant heterozygotes crossed into the appropriate wild-type background.

Elav-Gal4, cha-Gal4 (Salvaterra and Kitamoto, 2001), and UAS-GFP [P\{UAS-GFP.S65T\}T10] transgenic strains were obtained from the Drosophila Stock Center (Bloomington, IN). The TH-Gal4 strain was obtained from J. Hirsh (University of Virginia, Charlottesville, VA). UASGTPCH was generated by cloning the open reading frame of wild-type GTPCH cDNA into the P-element transformation vector ( $\mathrm{p} U A S T$ ) downstream of the UAS enhancer element. The resulting transformation vector was injected into $w^{1118}$ embryos by Genetic Services (Cambridge, $\mathrm{MA}$ ), and transgenic animals were isolated using established genetic methodology.

Paraquat, $\mathrm{H}_{2} \mathrm{O}_{2}, \mathrm{DA}, \mathrm{L}-\mathrm{DOPA}$, and 3-iodotyrosine exposure. Two slightly different methods were used for exposure of flies to paraquat and hydrogen peroxide. Separated adult male and female flies, $24-48 \mathrm{~h}$ after eclosion, were fed on filter paper saturated with $5 \%$ sucrose, or $5 \%$ sucrose containing 10 or $20 \mathrm{~mm}$ paraquat (Sigma, St. Louis, MO), or $5 \%$ sucrose containing $1 \%$ hydrogen peroxide. Alternatively, paraquat or hydrogen peroxide was mixed with $1 \%$ sucrose and $1.3 \%$ agar. These methods yielded comparable results. For monoamine or inhibitor studies, concentrations of L-DOPA, DA, and 3-iodotyrosine varied with the type of experiment and are noted in the legends of the figures. For mobility rescue experiments that were short term, these compounds were cofed with paraquat. For longer-term viability rescue, adults were prefed on the specified supplement for $72 \mathrm{~h}$ before exposure to paraquat.

Catalase assay. Flies at $24 \mathrm{~h}$ posteclosion were fed $20 \mathrm{~mm}$ paraquat in $5 \%$ sucrose or sucrose alone for 12 or $24 \mathrm{~h}$ before they were killed. Crude enzyme extracts were prepared by homogenizing 10 heads in $150 \mu \mathrm{l}$ of $0.1 \mathrm{M}$ sodium-potassium phosphate buffer, $\mathrm{pH} 7.0$, containing $0.1 \mathrm{M}$ Triton X-100 (Fisher Biotech, Hampton, NH) following the method of Bewley et al. (1983). After homogenization, all of the samples were incubated at $4^{\circ} \mathrm{C}$ for $10 \mathrm{~min}$ before centrifugation at $12,000 \times g$ for $10 \mathrm{~min}$. Assays were performed at $30^{\circ} \mathrm{C}$ following the methods of Lubinsky and Bewley (1979). The reaction was initiated by adding $20 \mu$ l of tissue extract to the reaction mixture containing $48.65 \mathrm{mM} \mathrm{H}_{2} \mathrm{O}_{2}$ in $0.1 \mathrm{M}$ sodiumpotassium phosphate buffer, $\mathrm{pH} 6.8$, to a final volume of $2.55 \mathrm{ml}$. The disappearance of $\mathrm{H}_{2} \mathrm{O}_{2}$ was monitored by following the decrease in ab- sorbance at $230 \mathrm{~nm}$ using a molar extinction coefficient for $\mathrm{H}_{2} \mathrm{O}_{2}$ of 62.4 (Nelson and Kiesow, 1972). One unit of catalase activity was defined as 1 mmol of $\mathrm{H}_{2} \mathrm{O}_{2}$ decomposed per minute. Specific activities are the averages of five to six replications derived from independently prepared extracts.

Locomotion assay. Movement deficits were assessed by deviation from normal negative geotaxis behavior. Ten adult flies per replication were placed in an empty plastic vial. After a $10 \mathrm{~min}$ rest period, the flies were tapped to the bottom of the vial, and the number of flies able to climb 5 $\mathrm{cm}$ in $60 \mathrm{~s}$ was recorded. The assays were repeated three times at $1 \mathrm{~min}$ intervals; the score for each replication was an average of the three time trials. Fifteen replications were performed for each data set.

Immunolocalization of dopaminergic neurons. Brains from $D f(1) w, y$ adults (paraquat treated and untreated) were fixed in $4 \%$ paraformaldehyde for $3.5 \mathrm{~h}$ and washed extensively in $1 \times \mathrm{PBS}$ and then in $0.1 \%$ Triton $\mathrm{X}-100,0.2 \%$ bovine serum albumin, in PBS (PBT). Brains were then blocked in $5 \%$ normal goat serum in PBT overnight at $4^{\circ} \mathrm{C}$, followed by overnight incubation with a 1:5000 dilution of rabbit anti-Drosophila $\mathrm{TH}$ antiserum. After additional washing, the brains were incubated at room temperature for $3 \mathrm{~h}$ in a 1:5000 dilution of $\mathrm{Cy}$-3-conjugated goat antirabbit IgG.

Confocal microscopy. Neurons were visualized in transgenic animals using the Gal4/UAS system to express green fluorescent protein (GFP) or immunostaining with anti-Drosophila $\mathrm{TH}$ antibody. Adults at $24-36 \mathrm{~h}$ posteclosion, expressing GFP under the control of TH-Gal4 (dopaminergic neuron expression), Chat-Gal4 (cholinergic neuron expression), or Elav-Gal4 (pan-neuronal expression), were fed $20 \mathrm{~mm}$ paraquat for various durations. Whole mounts of dissected brains were examined for dopaminergic neuron morphology and number using a Leica (Wetzlar, Germany) TCS SP2 AOBS confocal microscope.

HPLC analysis. Monoamines and pteridines were separated by HPLC analysis using a CoulArray HPLC system (model 5600A; ESA, Chelmsford, MA) and a Synergi $4 \mu \mathrm{m}$ Hydro-RP column $(4.6 \times 150 \mathrm{~mm}$; Phenomenex, Torrance, CA), according to the method of McClung and Hirsh (1999). Heads from 75 to 200 adults were extracted in 100-200 $\mu \mathrm{l}$ of $0.1 \mathrm{~m}$ perchloric acid; extracts were filtered through $0.2 \mu \mathrm{m}$ filters. Ten microliters of each extract were injected for each sample. The mobile phase contained 75 mu sodium phosphate, $\mathrm{pH}$ 3.0, 1.4 mM octanesulfonic acid, $25 \mu \mathrm{m}$ ethylene diamine tetraacetic acid, $100 \mu \mathrm{l} / \mathrm{L}$ triethylamine, and $7 \%$ acetonitrile. Separations were performed with isocratic flow at 1 $\mathrm{ml} / \mathrm{min}$. Amines were detected with an ESA electrochemical analytical cell (model 5011; channel 1 at $-50 \mathrm{mV}$, channel 2 at $300 \mathrm{mV}$ ). Pteridines were detected with a linear model LC305 fluorescence detector at excitation wavelength $360 \mathrm{~nm}$ and emission wavelength $465 \mathrm{~nm}$. Pool sizes were determined relative to freshly prepared standards (Sigma). Analysis was performed using ESA CoulArray software.

Statistical analysis. Analyses of all data were conducted using GraphPad (San Diego, CA) Prism, using two-tailed Student's $t$ test assuming equal variances, or using a one-way ANOVA followed by the Dunnett's post test. Details of analyses are described in the figure legends.

\section{Results}

\section{Paraquat-induced alteration in catalase activity}

The cellular pathology of paraquat has been extensively investigated. It has been shown to undergo intracellular reduction to a free radical form, which then is reoxidized in the presence of oxygen to initiate an oxidative cascade that includes the generation of superoxide radicals that are converted by superoxide dismutase to hydrogen peroxide, which is then used as a substrate for catalase (for review, see Dinis-Oliveira et al., 2006). Elevation of catalase expression in neural cell culture is reported to be an early cellular response to the onset of paraquat-generated oxidative stress (Rohrdanz et al., 2001). We therefore used catalase as a temporal indicator of the onset of an oxidative response. Assays of catalase activity in the heads of $24 \mathrm{~h}$ posteclosion adults fed on $20 \mathrm{~mm}$ paraquat for 12 and $24 \mathrm{~h}$ were compared with untreated male and female controls. The treatment and control sets were 

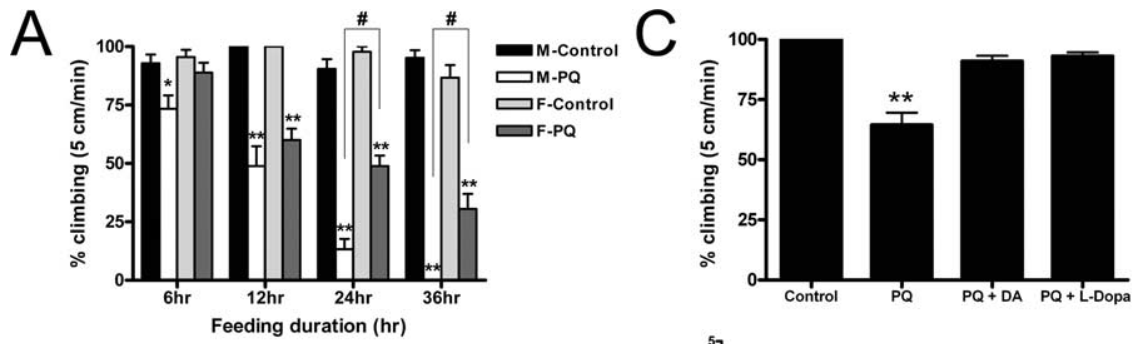

B

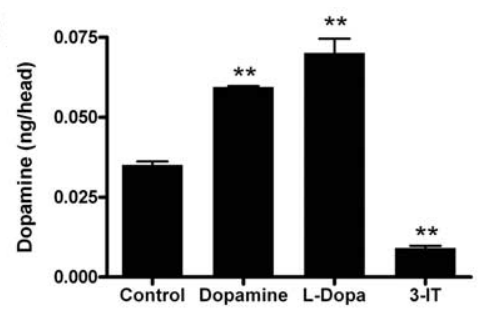

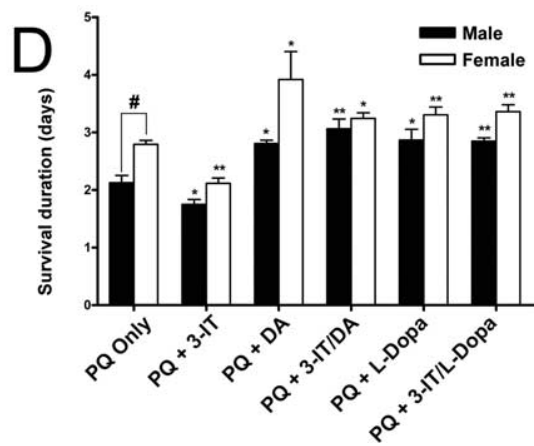

Figure 1. Effects of $20 \mathrm{~mm}$ paraquat on movement and life-span characteristics of wild-type Drosophila. $\boldsymbol{A}$, Negative geotaxis assays. The effect of paraquat on the mobility of adult males $(M)$ and females $(F)$ was determined by scoring the number of flies able to climb $5 \mathrm{~cm}$ in $60 \mathrm{~s}$, at 6, 12, 24, and $36 \mathrm{~h}$ after the initiation of ingestion of $20 \mathrm{~mm}$ paraquat. Ten flies were scored per replication, and the mean values represent the average of 15 independent replications. Loss of mobility in males was noted as early as $6 \mathrm{~h}$ after initiation of feeding, whereas effects on females were first noted by $12 \mathrm{~h}$. Male flies were completely immobile by $36 \mathrm{~h}$ of paraquat exposure. ${ }^{*} p<0.05,{ }^{* *} p<0.01$, significant differences between control and paraquat-fed flies; ${ }^{*} p<0.001$, significant differences in the effects of paraquat on males and females. $\boldsymbol{B}$, The pharmacological manipulation of DA pools. Adult flies were fed $0.5 \mathrm{~mm} \mathrm{DA}, 0.5 \mathrm{~mm}$ L-DOPA, or $10 \mathrm{~mm} 3$-iodotyrosine for $72 \mathrm{~h}$ after which DA pools in heads were determined, at the time period in which effects on mobility and survival were determined in parallel experiments. Both L-DOPA and DA ingestion significantly elevate DA pools, whereas ingestion of the TH inhibitor 3-iodotyrosine significantly depleted DA pools. ${ }^{* *} p<0.01$, significant differences. $C$, The rescue of mobility deficits by L-DOPA and DA. Adult males that were cofed L-DOPA (1 mm) or DA (1 mm) with paraquat $(20 \mathrm{~mm}$ ) were analyzed for mobility characteristics using the negative geotaxis assay. Cofeeding of paraquat with either DA or L-DOPA rescues paraquat-induced impairment of mobility. Cofeeding of paraquat with either L-DOPA or DA rescued mobility to levels that are near control mobility. ${ }^{* *} p<0.001$, differences between control and paraquat-fed flies. $D$, Effects of manipulation of DA pools on survival. Wild-type (Canton S) adults were fed DA (0.5 mm) or L-DOPA (0.5 mm) for $72 \mathrm{~h}$ before paraquat treatment to elevate DA levels, whereas the TH inhibitor 3-iodotyrosine was fed alone or with L-DOPA or DA at a concentration of $10 \mathrm{~mm}$ to deplete endogenous DA pools. The results show that pharmacological modulation of the DA pathway affected the life span, establishing that the effects of paraquat on life span are mediated through the dopaminergic pathway. Differences between male and female survival were tested for significance only in the paraquat-only sample; note that males are significantly more sensitive to paraquat as measured by average survival duration. ${ }^{*} p<0.001$, significant differences in the effects of paraquat on males and females. Each value is the average of $10-20$ replications, and each replication was made up of 10 flies. ${ }^{*} p<0.05,{ }^{* *} p<0.01$, significant difference between control and treated. Error bars indicate SEM. PQ, Paraquat; 3-IT, 3-iodotyrosine.

carefully age matched to account for the normal time-dependent reduction in catalase-specific activity that occurs during early posteclosion as the activity drops from a peak during pupation (Radyuk et al., 2000). The heads of control males had a catalasespecific activity of $3.49 \pm 0.08 \times 10^{6}$ units per milligram of protein at the $12 \mathrm{~h}$ assay point and $3.07 \pm 0.07 \times 10^{6}$ units per milligram of protein at $24 \mathrm{~h}$. Similarly, the heads of control females had a catalase-specific activity of $3.55 \pm 0.09 \times 10^{6}$ units per milligram of protein at $12 \mathrm{~h}$ and $3.02 \pm 0.12 \times 10^{6}$ units per milligram of protein at $24 \mathrm{~h}$. Extracts of body tissue (thorax and abdomen) had specific activities one order of magnitude higher than heads but also exhibited similar decreases in activity during the first $2 \mathrm{~d}$ after eclosion ( $p<0.001$, data not shown). Ingestion of paraquat resulted in highly significant elevations of catalase activity within $12 \mathrm{~h}$ in the heads of both males and females (supplemental Fig. 1, available at www.jneurosci.org as supplemental material). Relative to control activities, paraquat-fed males exhibited a $17.2 \%(p<0.003)$ increase, and females had a $14.4 \%$ increase $(p<0.007)$. At $24 \mathrm{~h}$ of paraquat exposure, catalase activity in the paraquat-treated males was $14.7 \%$ higher than control male activities $(p<0.03)$, whereas female heads sustained an increase in $23.5 \%$ by 24 h of exposure ( $p<$ $0.005)$. Differences in catalase activity between males and females were slight and not significant at any time point in this study. These results indicate that cellular responses to paraquat ingestion occur in both heads and bodies within $12 \mathrm{~h}$ of initial exposure.

\section{Paraquat exposure causes movement} disorders in adult Drosophila

Paraquat exposure has been linked to increased PD risk in agricultural communities (cf. Liou et al., 1997) and induces movement disorders in some mammalian models (Brooks et al., 1999; Chanyachukul et al., 2004). However, in other mammalian studies in which paraquatinduced dopaminergic neurodegeneration is observed, neuronal loss has not been sufficiently extensive to emulate PD movement characteristics (McCormack et al., 2002; Di Monte, 2003). Therefore, we assessed the effects of paraquat on mobility of adult flies. After exposure of wildtype adults to $20 \mathrm{~mm}$ paraquat, the flies exhibited rapid onset of movement disorders, including resting tremors, bradykinesis, rotational behaviors, and postural instability (supplemental videos 1 and 2, available at www.jneurosci.org as supplemental material), which mirror parkinsonian symptoms. Furthermore, the flies frequently froze while attempting to climb vial walls and would often fall to the bottom of the vial. Males exhibited symptoms $\sim 12$ h earlier than females, but both males and females were strongly affected. These results were quantified using a standard climbing assay based on the negative geotaxis behavior of adult flies. When tapped down to the bottom of a vial, adult flies right themselves and immediately climb up the sides of the vial. Within $6 \mathrm{~h}$ of 20 mM paraquat feeding, males were significantly slower than control males taken from the same cultures, and within $12 \mathrm{~h}$, the climbing abilities of both males and females were strongly incapacitated (Fig. $1 \mathrm{~A}$ ). However, consistent with the earlier onset of mobility deficits in males, paraquat ingestion affected males more severely than females ( $p<0.001$ at 24 and 36 h of exposure). Males exhibited near complete loss of mobility at $24 \mathrm{~h}$ and by $36 \mathrm{~h}$ had lost completely their ability to climb the $5 \mathrm{~cm}$ distance used in these assays. Females did not sustain deficits in mobility until $12 \mathrm{~h}$ of paraquat ingestion, and although they were strongly affected at 24 and $36 \mathrm{~h}$, they retained some capacity for movement, even at $36 \mathrm{~h}$ of exposure. Control flies climbed $5 \mathrm{~cm}$, on average, within 5-7 s, whereas flies exposed to paraquat for $24 \mathrm{~h}$ required, on average, $\sim 30$ s to climb this distance (regardless of sex) (data not shown).

We then asked whether the mobility deficit could be rescued by cofeeding wild-type adults with $1 \mathrm{~mm}$ L-DOPA or DA with paraquat for $24 \mathrm{~h}$. We first determined whether ingestion of ei- 
ther catecholamine could affect DA pools in heads and noted that both confer an approximate twofold increase in DA pools (Fig. $1 B$ ). Moreover, we detected no significant elevation in DA oxidation products in the HPLC chromatograms. Figure $1 C$ demonstrates the effects of supplementation on males; females were similarly affected (data not shown). Paraquat treatment alone resulted in poor mobility. Both L-DOPA and DA rescued the locomotor deficit to $85-90 \%$ of control levels. We also determined the average time required for flies to climb $5 \mathrm{~cm}$; samples fed paraquat only for $24 \mathrm{~h}$ required an average time of $\sim 25-30 \mathrm{~s}$ to move this distance compared with 5-7 s for untreated controls. Ingestion of either L-DOPA or DA with paraquat rescued mobility to an average time of $10 \mathrm{~s}$, near control mobility (data not shown). We conclude that paraquat ingestion induces a condition in this Drosophila strain that recapitulates the movement disorders of PD patients and the improvement of symptoms after L-DOPA therapy.

\section{Paraquat-induced reduction in life span can be modified by manipulation of DA pools}

Paraquat caused a concentration-dependent reduction in life span (supplemental Fig. 2, available at www.jneurosci.org as supplemental material). Males exposed to $20 \mathrm{~mm}$ paraquat had an average life span of $2 \mathrm{~d}$, whereas females survived 12-16 h longer (Fig. $1 D)(p<0.001)$. Decreasing the concentration of paraquat resulted in an extension of life span, but even at $100 \mu \mathrm{M}$, a dramatic reduction in survival was observed. We also noted that the movement disorders described above in $20 \mathrm{~mm}$ paraquat experiments preceded elevated mortality at all paraquat concentrations (data not shown).

We next investigated whether pharmacological manipulation of DA pools could alter the reduction in life span accompanying paraquat ingestion. DA depletion with 3-iodotyrosine caused wild-type flies to become more sensitive to paraquat, reducing male and female survival by $\sim 12-16 \mathrm{~h}$. In contrast, feeding either L-DOPA or DA to elevate the DA pools caused them to become significantly more tolerant to paraquat, increasing survival 12-24 $\mathrm{h}$, on average (Fig. 1D). Both L-DOPA and DA remain able to enhance paraquat tolerance in the presence of 3-iodotyrosine, confirming that the inhibitor does not cause nonspecific effects on paraquat tolerance. These results suggest that the modification of life span, as well as the movement disorders, are specifically related to effects on DA pools.

\section{Paraquat causes selective loss of DA neuron clusters}

To determine whether the observed movement disorders and reduced life span were associated with loss of DA neurons, neuronal survival was monitored using GFP expression driven by the TH promoter in TH-Gal4;UAS-GFP transgenic adults (FriggiGrelin et al., 2003). The neuronal clusters exhibiting GFP expression have been shown previously to be DA neurons by costaining with anti-TH antibody (Coulom and Birman, 2004). Figure $2 \mathrm{~A}$ illustrates the positions of each dopaminergic cluster scored. The numbers of dopaminergic neurons were clearly reduced as a consequence of paraquat ingestion (Fig. $2 B$ ). However, not all DA neuron clusters were simultaneously affected or affected to the same extent during paraquat exposure; rather, each cluster displayed distinctive and highly reproducible variation in sensitivity. For example, after only $6 \mathrm{~h}$ of exposure to paraquat, the protocerebral anterolateral clusters in the anterior region (Figs. $2 B, 3 A-F$ ) and the PPL1 cluster in the posterior region (Figs. $2 B$, $3 G-I, K)$ of the brain exhibited a significant reduction in neuron number relative to control brains, whereas none of the other clusters scored were affected during this period. By $12 \mathrm{~h}$, clusters PPM2 (Figs. $2 B, 3 G, H, J, L$ ) and PPM3 (Fig. $2 B$ ) were also losing neurons, whereas PPM1 and PPL2 showed significant neuronal loss only at 20-24 h exposure (Fig. $2 \mathrm{~B}$ ), when general neuronal damage becomes apparent (data not shown). Changes in neuronal morphology occurred in a highly predictable sequence, with the first evidence of the effects of paraquat being the retraction of neuronal processes, accompanied by distinct blebbing along many of the processes. As processes retract, cell bodies shift in position, tending to aggregate together (Fig. 3, compare $B, C$, controls with $E, F$, paraquat treated). The cell bodies became rounded, subsequently fragment, and finally disappear. Examples of these changes are indicated by arrowheads; control neurons are indicated by arrows (Fig. 3). These results were confirmed in brains immunostained with anti-Drosophila $\mathrm{TH}$ antibody (supplemental Fig. 3, available at www.jneurosci.org as supplemental material); no significant differences were observed between GFP reporter and immunostained brains. The temporal progression of neural degeneration coincided quite closely with the timing of symptom onset and progression of movement deficits.

The specificity of paraquat-induced neurodegeneration was established in several ways. First, we examined the effects of paraquat on cholinergic neurons using the promoter of the choline acetyltransferase gene to drive GFP expression (Salvaterra and Kitamoto, 2001) (Fig. 4A-D). These neurons are organized in larger clusters than the dopaminergic neurons, and it was therefore difficult to obtain highly reproducible counts of cholinergic neurons. However, in most regions, the neurons were sufficiently distinct for an examination of neuronal morphology during paraquat exposure. We were unable to detect any neurons exhib- 

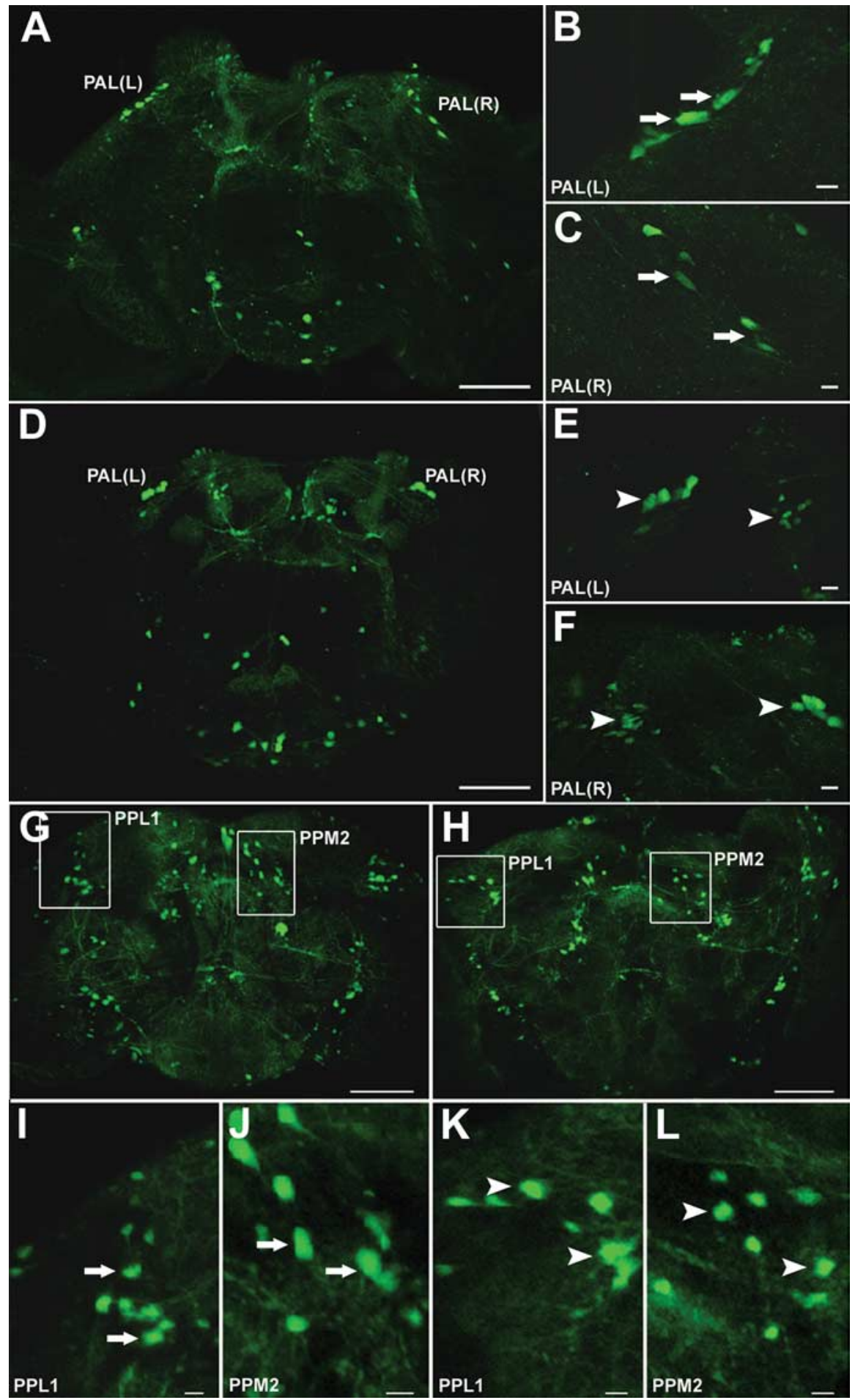

Figure 3. Effect of paraquat on neuronal survival. $\boldsymbol{A}-\boldsymbol{F}$, The effect of $6 \mathrm{~h}$ of $20 \mathrm{~mm}$ paraquat ingestion on anterior DA cluster PAL in w;TH-Gal4;UAS-GFP adult brain. Arrows highlight the asymmetrical shape of control neurons with extended processes, whereas arrowheads designate neurons altered by exposure to paraquat. $A$, Control brain. $B, C$, Enlarged views of the PAL clusters (left and right, respectively) of the dopaminergic neurons of the brain in $\boldsymbol{A}$. $\boldsymbol{D}$, Paraquat-treated brain. $\boldsymbol{E}, \boldsymbol{F}$, Enlarged views of paraquatexposed PAL clusters (left and right, respectively) in $\boldsymbol{D}$, demonstrating rapid loss of cells and aggregation of remaining cells caused by the retraction of neuronal processes, as indicated by the arrowheads. The left arrowhead in $\boldsymbol{F}$ shows neurons in the process of fragmenting. $\mathbf{G}-\mathbf{L}$, Effect of $12 \mathrm{~h}$ exposure to $20 \mathrm{~mm}$ paraquat on posterior dopaminergic clusters in w;TH-Gal4;UAS-GFP adult brain. $\boldsymbol{G}, \boldsymbol{H}$, Control $(\boldsymbol{G})$ and paraquat-treated $(\boldsymbol{H})$ brains. White boxes indicate DA neuron clusters PPL1 and PPM2, which are enlarged in $\boldsymbol{I}-\boldsymbol{L}$. $\boldsymbol{I}$, Control PPL1. J, Control PPM2. $\boldsymbol{K}$, Paraquat-exposed PPL1. L, Paraquat-exposed PPM2. Both PPL1 and PPM2 clusters exhibit loss of cells and rounding of remaining neurons in paraquat-treated brains (arrowheads) compared with control neurons (arrows). Scale bars: $\boldsymbol{A}, \boldsymbol{D}, \boldsymbol{G}, \boldsymbol{H}, 100 \mu \mathrm{m} ; \boldsymbol{B}, \boldsymbol{C}, \boldsymbol{E}, \boldsymbol{F}, \boldsymbol{I}-\boldsymbol{L}, 50 \mu \mathrm{m}$. iting process retraction, cell rounding, or areas of clear reduction in neuron numbers. We also examined brains expressing GFP driven by the pan-neuronal promoter Elav to determine whether paraquat was universally detrimental to CNS neurons. Although the effect of paraquat on DA neurons varies among clusters, we observe an overall reduction in neuron numbers in dopaminergic neurons on the order of $30-50 \%$ by $12 \mathrm{~h}$ of exposure. We reasoned that if paraquat had a similar effect on all neurons, we would be able to detect a $30-50 \%$ overall reduction in neuron numbers. The general morphology of the brain and neuron viability were unaffected through the first $12 \mathrm{~h}$ of paraquat ingestion (Fig. 4E,F); more generalized neuronal death becomes apparent by $24 \mathrm{~h}$ of exposure, when all dopaminergic clusters become affected (data not shown).

We then examined the effect of overexpressing GTPCH via a UAS-GTPCH transgene specifically in dopaminergic cells using TH-Gal4. We reasoned that because GTPCH is rate limiting for the synthesis of $\mathrm{BH}_{4}$ in Drosophila (Krishnakumar et al., 2000) and the cofactor, in turn, is limiting for DA synthesis, overexpression should elevate both cofactor and DA levels. The pharmacological analyses described above led to the prediction that DA overexpression should lead to greater resistance to the effects of paraquat if its effects were initially limited to dopaminergic cells. As shown in Figure 5, these expectations were met. Wild-type controls, UAS-GTPCH transgenic flies in the absence of Gal4 expression, and $\mathrm{TH}$ Gal4 transgenic flies had an average survival time of 3-4 d with ingestion of 10 $\mathrm{mM}$ paraquat. Transgenic flies with both the TH-Gal4 and UAS-GTPCH transgenes survived $\sim 24 \mathrm{~h}$ longer than the controls.

Finally, we sought to determine whether the neurodegenerative action of paraquat was an example of a generalized response to oxidative stressors or an indicator of specific access of paraquat to dopaminergic neurons. Wild-type adults were fed $1 \%$ hydrogen peroxide for a period of $60 \mathrm{~h}$, at which time mortality had reached at least 50\%. Although the treatment was lethal to these flies, at no time did they exhibit neurological syndromes. Moreover, examination of dopaminergic neuron numbers and morphology (supplemental Fig. 4, available at www.jneurosci.org as supplemental material) revealed no loss of neurons and complete absence of morphological alterations. 
These four experiments in combination lead to the conclusion that dopaminergic neurons are uniquely and specifically at risk during paraquat exposure.

\section{Effect of paraquat on the DA and $\mathrm{BH}_{4}$ pathways}

The results described above closely link the deleterious effects of paraquat to dopaminergic function in Drosophila. This conclusion leads to the prediction that it should be possible to observe effects on the biosynthetic products and metabolites of the DA pathway in adult heads early in the period of paraquat exposure. Moreover, because $\mathrm{BH}_{4}$ is required for and modulates DA synthesis, and $\mathrm{BH}_{4}$ synthesis is coregulated with DA pathway activity (Levine et al., 1981; Sumi-Ichinose et al., 2005), parallel changes would be expected in the $\mathrm{BH}_{4}$ biosynthesis pathway. We therefore examined the effect of paraquat ingestion on the pool sizes of intermediates and metabolites of the $\mathrm{BH}_{4}$ and DA pathways. In both males and females, elevation of L-DOPA levels during the feeding period was noted (Fig. 6A). Because L-DOPA is the immediate product of $\mathrm{TH}$ catalysis, this result indicates a stimulation of TH activity. However, in males, significant loss of DA, produced by decarboxylation of L-DOPA, was observed by $6 \mathrm{~h}$ of paraquat ingestion, and by $12 \mathrm{~h}$, DA pools in both males and females were diminished (Fig. 6B). On the other hand, DOPAC, which is virtually undetectable in adult Drosophila brains under normal conditions until $48 \mathrm{~h}$ after eclosion, was strongly elevated after paraquat exposure (Fig. 6C). Together with the observation of enhanced L-DOPA production, these results suggest that paraquat exposure not only stimulates the biosynthetic pathway but also leads to increased accessibility of DA to oxidation. Similarly, a concomitant and strong elevation in biopterin, the fully oxidized derivative of $\mathrm{BH}_{4}$, suggests a compensatory increase in GTPCH activity, although $\mathrm{BH}_{4}$ seems to be highly sensitive to the elevated oxidative conditions in neurons exposed to paraquat (Fig. $6 D, E$ ). It is interesting to note that females have a higher content of both L-DOPA and DA compared with males, coinciding with gender differences in paraquat susceptibility (Fig. 6 A,B)

\section{Differential effects of paraquat on DA-regulatory mutants}

The strong effects of paraquat on the $\mathrm{BH}_{4}$ and DA pathways and the consequences of pharmacological modulation of DA pools on paraquat-induced damage led to the hypothesis that mutations in genes that are part of the DA homeostatic network would affect tolerance to the herbicide. Mutations in three genes were examined: ple, which encodes $\mathrm{TH}$, the rate-limiting enzyme in the DA biosynthesis pathway; $\mathrm{Pu}$, which encodes $\mathrm{GTPCH}$, the ratelimiting enzyme in the synthesis of $\mathrm{BH}_{4}$; and Catsup, a negative regulator of DA synthesis, which causes overproduction of DA in loss-of-function mutants (Stathakis et al., 1999). Because mutations in these genes are homozygous lethal, all strains investigated were necessarily heterozygous with a wild-type allele of each gene.

We confirmed that alterations in the activities of $\mathrm{TH}$ and GTPCH resulted in concomitant changes in $\mathrm{DA}$ and $\mathrm{BH}_{4}$ pools by HPLC analysis of extracts prepared from the heads of representative mutant strains (Fig. 7). Because TH requires $\mathrm{BH}_{4}$ for activity, loss-of-function mutations in $\mathrm{Pu}$, as the rate-limiting step in $\mathrm{BH}_{4}$ synthesis, should affect both cofactor and DA pools. As expected, the mutant allele $\mathrm{Pu}^{\mathrm{Z22}}$ caused loss of both $\mathrm{BH}_{4}$ (Fig. $7 A$ ) and DA (Fig. 7B). The mutant allele ple $e^{2}$ caused diminished DA pools (Fig. $7 B$ ) but no significant reduction in $\mathrm{BH}_{4}$ (Fig. 7A). Catsup mutations resulted in strongly elevated $\mathrm{BH}_{4}$ (Fig. 7A) and DA (Fig. 7B) levels.

Having established (1) that DA neurons are indeed at risk 


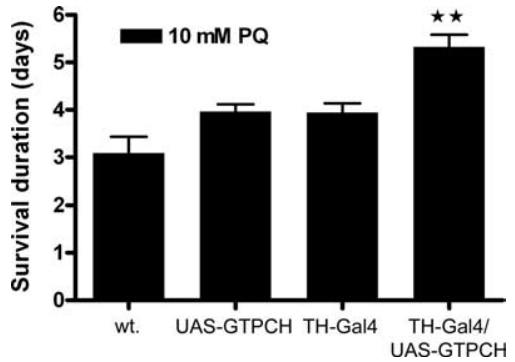

Figure 5. Overexpression of GTPCH in dopaminergic neurons increases resistance to paraquat. Wild type (wt.) $[D f(1) w, y]$ and the transgenic strains UAS-GTPCH (without the Gal 4 driver) and $T H$-Gal4 (without the UAS promoter element) had equivalent average survival durations when exposed to $10 \mathrm{~mm}$ paraquat $(\mathrm{PQ})$. Female flies in which GTPCH was overexpressed specifically in dopaminergic neurons using the $\mathrm{TH}$-Gal 4 driver survived nearly $24 \mathrm{~h}$ longer than controls when exposed to paraquat. Males were similarly affected (data not shown). Error bars indicate SEM. The GTPCH overexpression flies were more resistant at ${ }^{* *} p<0.01$.

viving as much as $3 \mathrm{~d}$ longer than either wild-type strain (Fig. 7C). In contrast, $p l e^{2}$ and $P u^{Z 22}$ heterozygotes exhibited reduced tolerance to paraquat, surviving for as little as $24 \mathrm{~h}$. These results support the hypothesis that variation in DA-regulating genes can alter susceptibility to dopaminergic neuron-specific damage. In theory, differential sensitivity to paraquat in these mutants could be attributable either to alteration in DA pools per se or to the morphological or functional consequences of having altered DA production throughout development. Because it was possible to phenocopy pharmacologically the modified stress tolerance of the mutants by feeding the inhibitor 3-iodotyrosine to adults when neural development is complete, we conclude that differential susceptibility of the heterozygous mutants to paraquat is directly attributable to alterations in the DA pool in the adult flies and does not result from modification of neural development.

We next compared the effects of $1 \%$ hydrogen peroxide on wild-type adults and Catsup and $P u$ mutants (Fig. 7D). Interestingly, Catsup flies were more sensitive than wild-type flies to hydrogen peroxide, whereas the survival of the $P u$ mutant strain was indistinguishable from wild-type survival. Thus, despite the absence of neurodegenerative effects of $\mathrm{H}_{2} \mathrm{O}_{2}$ ingestion (supplemental Fig. 4 , available at www.jneurosci.org as supplemental material), sensitivity to this oxidative compound is clearly affected by mutations in the Catsup gene. This result lessens the possibility that the high DA levels in Catsup mutant strains are affecting resistance to paraquat via enhanced cuticlular cross-linking that might form a stronger barrier to oxidative compounds entering the body directly rather than through ingestion.

\section{Neuroprotective effects of Catsup mutations}

Several possible mechanisms could be responsible for the enhanced survival and later onset of mobility deficits in Catsup mutants. Because we observe increased catalase activity in paraquat-exposed, wild-type adults, one potential mechanism could be that Catsup mutations might have an additional phenotype, an elevation in the specific activity of catalase. Increased catalase activity would be expected to increase scavenging capacity. However, assays of catalase in the Catsup $p^{26}$ mutant and a wild-type control demonstrated that the specific activities of catalase in these two strains were indistinguishable (supplemental Fig. 5, available at www.jneurosci.org as supplemental

during paraquat exposure of wild-type Drosophila, (2) that survival and movement deficits could be modified by DA and L-DOPA supplementation, and (3) that both the $\mathrm{BH}_{4}$ and $\mathrm{DA}$ pathways respond to paraquat, we next tested the hypothesis that variation in these genes, which are directly involved in DA homeostasis, could modify susceptibility to paraquat-induced oxidative stress. Heterozygous strains carrying mutant Catsup alleles exhibited strongly enhanced tolerance to $20 \mathrm{~mm}$ paraquat, sur- material). Another possible basis for the delayed onset of paraquat damage might be the elevated $\mathrm{DA}$ or $\mathrm{BH}_{4}$ pools resulting from loss of the negative regulatory function of Catsup. Increased pools in the brain could offset the effects of neuron loss, by maintaining the DA-dependent functions necessary for survival for longer periods than wild-type controls. Alternatively, the mutations could be preventing neuron loss. To address this question, we crossed the Catsup ${ }^{26}$ mutation into a TH-Gal4;UAS-GFP 
transgenic strain and compared the numbers of neurons in each cluster with those in the TH-Gal4;UAS-GFP control. The Catsup mutation itself had no effect on the number of DA neurons at $24 \mathrm{~h}$ after eclosion, under control conditions (Fig. $8 \mathrm{~A}$ ). However, ingestion of $20 \mathrm{~mm}$ paraquat for 24 h by wild-type and Catsup mutant heterozygotes resulted in a dramatic difference in the number of DA neurons in each cluster (Fig. $8 \mathrm{~B}$ ). After $24 \mathrm{~h}$ of paraquat exposure, the neurons in every dopaminergic cluster in wild-type brains exhibited extensive neurodegeneration. Strikingly, there was only a slight reduction in the number of neurons in a subset of DA clusters in Catsup $^{26} /$ Catsup $^{+}$brains under these conditions. For example, the PPM2 cluster in Catsup brains had approximately nine neurons in untreated brains (Fig. $8 \mathrm{~A}$ ) and an average of seven neurons after 24 h of paraquat exposure (Fig. $8 B$ ). The number of neurons in this cluster in untreated wild type brains was identical to the number in Catsup mutant brains (Fig. $8 A$ ), but after the 24 h exposure to paraquat, fewer than five neurons survived in the wild-type PPM2 cluster (Fig. 8B). Moreover, in other clusters, most notably PPM1 and PPL2, where paraquat-induced loss of neurons in wild type was severe, there were no significant differences in neuron numbers in control and paraquattreated Catsup ${ }^{26} /$ Catsup $^{+}$brains. Thus, we conclude that the reduction in functional Catsup protein serves a neuroprotective role against paraquatinduced oxidative stress.

\section{Discussion}

The Drosophila system described here robustly models environmental toxin-induced parkinsonism. Our methods result in a neurodegenerative response to paraquat in adult Drosophila, induced by oxidative stress, that mirrors most behavioral features of $\mathrm{PD}$, including resting tremors, bradykinesis, and postural instability. In addition, dopaminergic neurons are targeted during the early stages of oxidative damage. As in $\mathrm{PD}$, neurodegeneration exhibited regional differences, with particular neuronal groups responding early, and degenerating before DA neurons in other regions exhibited signs of damage, comparable to the preferential degeneration of nigral dopaminergic neurons in PD. Interestingly, these effects appear to be paraquat specific, in that another oxidative stressor, hydrogen peroxide, induced no movement disorders and no neuron loss, at exposure times that compromised viability. Finally, we observed that male Drosophila are more susceptible than females to paraquat at the ages used for this study, developing earlier mobility deficits and succumbing earlier. This reflects epidemiological studies of PD, which consistently report incidence rates in males 1.5-2.5 times those in females (Strickland and Bertoni, 2003; Van Den Eeden et al., 2003). Although the basis for gender differences is not understood, its occurrence in this model system, which was also noted by Neckameyer and Weinstein (2005), paves the way for genetic dissection of this phenomenon.

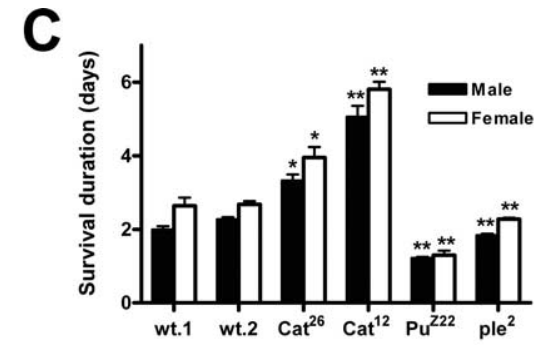

D

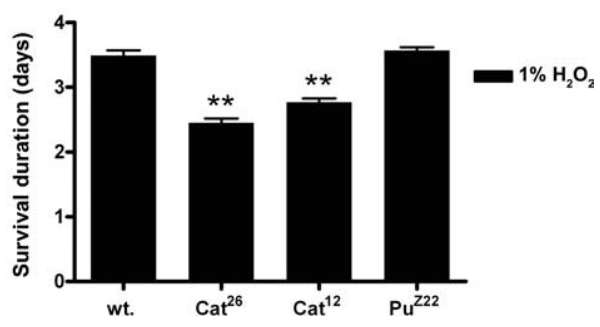

Figure 7. $\quad \boldsymbol{A}, \boldsymbol{B}$, Effect of $\mathrm{DA}$ pathway mutations on $\mathrm{BH}_{4}$ and $\mathrm{DA}$ in adult heads. Extracts were prepared from $48 \mathrm{~h}$ posteclosion adults. Each value is the average of $5-10$ replications, and each replication is made up of 150-200 pooled heads. $A$, Effect of 作

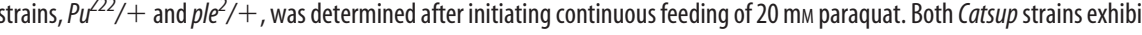
strain was indistinguishable from the wild type in its sensitivity to hydrogen peroxide. wt., Df(1)w; Cat ${ }^{26}$, w; Catsup $^{26} /+;$ Cat $^{12}$, w; Catsup $^{12} /+; \mathrm{Pu}^{\mathrm{Z22}}$, w; $\mathrm{Pu}^{722} /+;$ ple $^{2}$, w; $\mathrm{Ple}^{2} /+$.

We tested the effects of heterozygous mutations in DAregulating genes and found variation in the sensitivity of mutants to paraquat affecting the timing of symptom onset and life span.

Higher DA production levels enhance tolerance, whereas DA deficits resulted in sensitivity, to paraquat. Pharmacological treatment with L-DOPA rescued mobility deficits, another parallel to human PD. Unlike human PD patients, however, Drosophila movement disorders also responded to DA. DA does not transit the blood-brain barrier of mammals, but apparently there is no comparable blockage of DA in flies because its ingestion resulted in elevated DA pools in adult heads that influence movement and survival. In contrast, depletion of DA with a TH inhibitor increased paraquat sensitivity. The ability to phenocopy mutant effects pharmacologically after cessation of development establishes that variation in paraquat susceptibility is not caused by developmental effects of the mutations. Last, we observed that mutations in a negative regulator of $\mathrm{BH}_{4}$ and DA synthesis, Catsup, which might act through extension of mobility function and therefore prolonged feeding capability, confer dominant neuroprotection during exposure to paraquat. The results reported here mirror those of McCormack et al. (2002) in their study of the effects of systemic paraquat exposure in mice. In addition to regionally specific loss of dopaminergic neurons in mice and in these Drosophila strains, we also observe a similar compensatory response in which DA biosynthesis is stimulated as neuronal loss is initiated. Behavioral alterations in these flies also parallel mouse PD models, particularly hypoactivity (Brooks et al., 1999) 

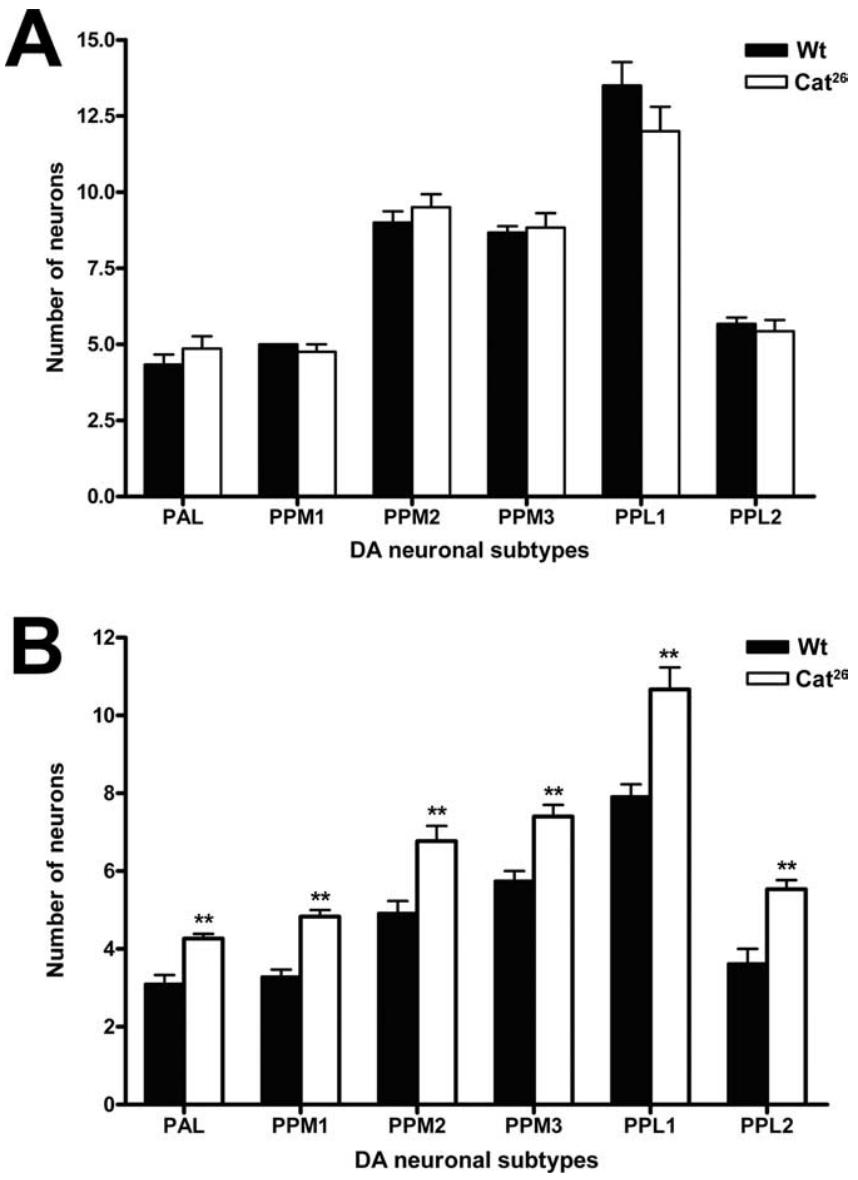

Figure 8. Dominant neuroprotection of Catsup mutations. $A$, Comparison of the numbers of dopaminergic neurons in Df(1)w;+/TH-Gal4;+/UAS-GFP and w; (atsup ${ }^{26} / \mathrm{TH}-G a l 4 ;+/ U A S-$ GFP adult brains from flies fed on sucrose only. No significant differences between Catsup mutant and wild-type brains were found in the number of neurons in any dopaminergic neuron cluster. $\boldsymbol{B}$, Comparison of the numbers of dopaminergic neurons in Df(1)w; +/TH-Gal4; + UAS GFP and w; Catsup $26 / T H-G a l 4 ;+/ U A S-G F P$ adult brains from flies fed on 20 mm paraquat for $24 \mathrm{~h}$. ${ }^{* *} p<0.01$, significant difference between wild-type and Catsup mutant neurons. For each set of samples, neurons from 15-20 brains were examined. Error bars indicate SEM. Wt, Wild-type.

and rotational behaviors (Chanyachukul et al., 2004). Motor deficits were also noted in Drosophila DJ-1 mutants after paraquat exposure (Park et al., 2005). We have extended the Drosophila model in that our procedures elicit a wide spectrum of PD behavioral characteristics.

It should be noted that in another report of paraquat effects in Drosophila hypersensitivity of DJ-1 mutants to oxidative stressors, no specific loss of dopaminergic neurons nor movement disorders were observed (Meulener et al., 2005). On the other hand, our results parallel those of Coulom and Birman (2004), who used rotenone as an oxidative stressor in another Drosophila model. Furthermore, there is a striking similarity between our results and those of Menzies et al. (2005), who reported that loss-of-function $D J-1 \beta$ mutants (a model for familial PD) or DJ-1 $\alpha$ overexpressed in dopaminergic neurons exhibit resistance to paraquat and protection of dopaminergic neurons in the adult brain, as we observe in loss-of-function Catsup mutants and transgenic adults overexpressing GTPCH in DA neurons. Interestingly, both $D J-1 \beta$ and Catsup mutants are sensitive to $\mathrm{H}_{2} \mathrm{O}_{2}$. It is not known whether the products of Catsup and DJ-1 functionally interact; this question will be an interesting avenue for future studies.

The basis for the reported differences in the Drosophila models described above is unknown but is likely to reflect both genetic background and environmental differences. These insect systems, like mammalian models (Di Monte, 2003), demonstrate the variability in responses that has made investigating the etiology of sporadic $\mathrm{PD}$ a major challenge.

The parallel results for $D J-1$ and Catsup mutants support our interpretation that the focal point of the paraquat response is neural and unrelated to other roles of DA such as cross-linking of cuticle, which potentially could affect the movement of paraquat into the body directly through contact rather than through the digestive tract. Because most of our assays have used entire heads rather than dissected brains, it is a formal possibility that variation in cuticular DA might affect our results, particularly in light of the results of Hardie and Hirsh (2006), who found a significant difference between the DA content of entire heads and dissected brains. We have, however, been unable to extract DA from dissected cuticle and underlying hypoderm from flies $24-72 \mathrm{~h}$ posteclosion (the age we used in all experiments), by which time cuticular differentiation is essentially complete (our unpublished observations). The fact remains that we see dopaminergic neuron loss within $6 \mathrm{~h}$ of exposure to paraquat, and this neuronal toxicity precedes the onset of neurological symptoms.

Our finding that mutations and pharmacological treatments that lower DA pools increase sensitivity to paraquat, whereas those that elevate DA provide protection, is surprising in light of extensive research revealing that DA contributes to oxidative load (Stokes et al., 1999) and our corresponding observation that Catsup mutants increase sensitivity to $\mathrm{H}_{2} \mathrm{O}_{2}$. These results suggest a model in which paraquat, which is highly similar in structure to $\mathrm{MPP}^{+}$(Dinis-Oliveira et al., 2006), has unique access to dopaminergic neurons that could be modified by competition with extracellular DA. The mechanism by which paraquat confers selective damage to dopaminergic neurons, however, is unresolved, and although evidence demonstrating the effects of DA reuptake inhibitors on paraquat toxicity supports specific access to DA neurons (Shimizu et al., 2003), definitive evidence is lacking. Our results suggest that this Drosophila model may be an ideal system in which to test such models.

The Catsup mutants also coordinately elevate $\mathrm{BH}_{4}$ pools. The tight integration of $\mathrm{DA}$ and $\mathrm{BH}_{4}$ responses in mutants and wildtype strains makes separation of roles difficult. However, $\mathrm{BH}_{4}$ has been implicated in many functions, in addition to DA production, which may also contribute to the enhancement of protection against paraquat in Catsup mutants. Elevation of intracellular $\mathrm{BH}_{4}$ via uptake of its precursor, sepiapterin, was reported to protect neurons in primary cultures of substantia nigra slices against the toxicity of $\mathrm{MPP}^{+}$(Madsen et al., 2003) and in dopaminergic neuron cultures subjected to oxidative stress by depletion of glutathione (Nakamura et al., 2001). Moreover, $\mathrm{BH}_{4}$ serves as an antioxidant in preventing nitric oxide toxicity in endothelial cells (Shimizu et al., 1998). In such cases, $\mathrm{BH}_{4}$ is able to scavenge ROS induced by paraquat. In its capacity as a scavenger, it could prevent the oxidation of DA, which we monitor as dramatically increased pools of DOPAC in the heads of adult flies exposed to paraquat (Fig. 1C).

Our finding that elevated DA pools provide oxidative protection against paraquat-induced toxicity is in apparent disagreement with the finding that overexpression of $\mathrm{TH}$ in dopaminergic neurons in Caenorhabditis elegans results in hypersensitivity to oxidative stress (Cao et al., 2005). Two factors may contribute to these differences. There is generally a requirement for integrated responses of the $\mathrm{BH}_{4}$ and DA biosynthesis pathways. Haavik et al. (1997) reported that uncoupling of $\mathrm{BH}_{4}$ oxidation and L-tyrosine 
hydroxylation can contribute to oxidative load. When $\mathrm{TH}$ is overexpressed, in the absence of coexpression of GTPCH, uncoupling is likely as the ratio of oxidative states of the cofactor shifts strongly to various oxidized states as TH protein levels increase. Moreover, the DA that is generated by TH overexpression may not be elevating concomitant release of the transmitter.

It should be noted that $\mathrm{BH}_{4}$ is neurotoxic in one rat model in which it was administered directly into the striatum, causing reduction in DA pools and loss of TH immunoreactivity (Kim et al., 2003). These results agree with cell-culture models from the same laboratory in which cytotoxicity induced by $\mathrm{BH}_{4}$ in the culture medium was dependent on the presence of intracellular DA (Choi et al., 2003). In both the cell-culture and rat models, toxicity is associated with high levels of extracellular $\mathrm{BH}_{4}$, which leads to compromised membranes; these conditions could reflect those of parkinsonism in later stages of the disease, in which oxidative damage to membranes could allow extracellular leakage of $\mathrm{BH}_{4}$.

We have reported here evidence that variation in genes, conserved in humans but not known to be associated with hereditary $\mathrm{PD}$, can alter susceptibility to a known environmental PD risk factor. These data serve as a proof of principle demonstrating that the system we have developed can be used to efficiently test putative PD-susceptibility genes. We also note that it represents a system that should be well suited to the testing of potential neuroprotective therapeutics.

\section{References}

Betarbet R, Sherer TB, Mackenzie G, Garcia-Osuna M, Panov AV, Greenamyre JT (2000) Chronic systemic pesticide exposure reproduces features of Parkinson's disease. Nat Neurosci 3:1302-1306.

Bewley GC, Nahmias JA, Cook JL (1983) Developmental and tissue-specific control of catalase expression in Drosophila melanogaster: correlations with rates of enzyme synthesis and degradation. Dev Genet 4:49-60.

Bilen J, Bonini NM (2005) Drosophila as a model for human neurodegenerative disease. Annu Rev Genet 39:153-171.

Brooks AI, Chadwick CA, Gelbard HA, Cory-Slechta DA, Federoff HJ (1999) Paraquat-elicited neurobehavioral syndrome caused by dopaminergic neuron loss. Brain Res 823:1-10.

Cao S, Gelwix CC, Caldwell KA, Caldwell GA (2005) Torsin-mediated protection from cellular stress in the dopaminergic neurons of Caenorhabditis elegans. J Neurosci 25:3801-3812.

Carbone MA, Jordan KW, Lyman RF, Harbison ST, Leips J, Morgan TJ, DeLuca M, Awadalia P, Mackay TFC (2006) Phenotypic variation and natural selection at Catsup, a pleiotropic quantitative trait in Drosophila. Curr Biol 16:912-919.

Chanyachukul T, Yoovathaworn K, Thongsaard W, Chongthammakum S, Navasumrit P, Satayavivad J (2004) Attenuation of paraquat-induced motor behavior and neurochemical disturbances by $\mathrm{L}$-valine in vivo. Toxicol Lett 150:251-269.

Choi HJ, Kim SW, Lee SY, Hwang O (2003) Dopamine-dependent cytotoxicity of tetrahydrobiopterin: a possible mechanism for selective neurodegeneration in Parkinson's disease. J Neurochem 86:143-152.

Cooper AA, Gitler AD, Cashikar A, Haynes CM, Hill KJ, Bhullar B, Liu K, Xu K, Strathearn KE, Liu F, Cao S, Caldwell KA, Caldwell GA, Marsischky G, Kolodner RD, LaBaer J, Rochet J-C, Bonini NM, Lindquist S (2006) $\alpha$-Synuclein blocks ER-Golgi traffic and Rab1 rescues neuron loss in Parkinson's models. Science 313:324-328.

Coulom H, Birman S (2004) Chronic exposure to rotenone models sporadic Parkinson's disease in Drosophila melanogaster. J Neuronsci 24:10993-10998.

De Bellis MD, Baum AS, Birmaher B, Keshavan MS, Eccard CH, Boring AM, Jenkins FJ, Ryan ND (1999) Developmental traumatology, part I: Biological stress systems. Biol Psychiatry 45:1259-1270.

Di Monte DA (2003) The environment and Parkinson's disease: is the nigrostriatal system preferentially targeted by neurotoxins? Lancet Neurol 2:531-538.
Dinis-Oliveira RJ, Remião F, Carmo H, Duarte JA, Sánchez Navarro A, Bastos ML, Carvalho F (2006) Paraquat exposure as an etiological factor of Parkinson's disease. NeuroToxicol 27:1110-1122.

Friggi-Grelin F, Coulom H, Meller M, Gomez D, Hirsh J, Birman S (2003) Targeted gene expression in Drosophila dopaminergic cells using regulatory sequences from tyrosine hydroxylase. J Neurobiol 54:618-627.

Haavik J, Almås B, Flatmark T (1997) Generation of reactive oxygen species by tyrosine hydroxylase: a possible contribution to the degeneration of dopaminergic neurons? J Neurochem 68:328-332.

Hardie SL, Hirsh J (2006) An improved method for the separation and detection of biogenic amines in adult Drosophila brain extracts by high performance liquid chromatography. J Neurosci Methods 153: 243-249.

Kim ST, Choi JH, Chang JW, Kim SW, Hwang O (2005) Immobilization stress causes increase in tetrahydrobiopterin, dopamine, and neuromelanin and oxidative damage in the nigrostriatal system. J Neurochem 95:89-98.

Kim WK, Jang YL, Chang JW, Hwang O (2003) Degeneration of the nigrostriatal pathway and induction of motor deficit by tetrahydrobiopterin: an in vivo model relevant to Parkinson's disease. Neurobiol Dis 13:167-176.

Krishnakumar S, Burton D, Rasco J, Chen X, O’Donnell J (2000) Functional interactions between GTP cyclohydrolase I and tyrosine hydroxylase in Drosophila. J Neurogenet 14:1-23.

Kumer SC, Vrana KE (1996) Intricate regulation of tyrosine hydroxylase activity and gene expression. J Neurochem 67:443-462.

Langston JW, Ballard P, Tetrud JW, Irwin I (1983) Chronic Parkinsonism in humans due to a product of melperidine-analog synthesis. Science 219:979-980.

Levine RA, Miller LP, Lovenburg W (1981) Tetrahydrobiopterin in striatum: localization in dopamine nerve terminals and role in catecholamine synthesis. Science 214:919-921.

Liou HH, Tsai MC, Chen CJ, Jeng JS, Chang YC, Chen SY, Chen RC (1997) Environmental risk factors and Parkinson's disease: a case-control study in Taiwan. Neurology 48:1583-1588.

Lubinsky S, Bewley GC (1979) Genetics of catalase in Drosophila melanogaster: rates of synthesis and degradation of the enzyme in flies aneuploid and euploid for the structural gene. Genetics 91:723-749.

Mackay W, O'Donnell JM (1983) A genetic analysis of the pteridine biosynthetic enzyme, guanosine triphosphate cyclohydrolase, in Drosophila melanogaster. Genetics 105:35-53.

Madsen JT, Jansen P, Hesslinger C, Meyer M, Zimmer J, Gramsbergen B (2003) Tetrahydrobiopterin precursor sepiapterin provides protection against neurotoxicity of 1-methyl-4-phenylpyridinium in nigral slice cultures. J Neurochem 85:214-223.

McClung C, Hirsh J (1999) The trace amine tyramine is essential for sensitization to cocaine in Drosophila. Curr Biol 9:853-860.

McCormack AL, Thiruchelvam M, Manning-Bog AB, Thiffault C, Langston JW, Cory-Slechta DA, Di Monte DA (2002) Environmental risk factors and Parkinson's disease: selective degeneration of nigral dopaminergic neurons caused by the herbicide paraquat. Neurobiol Dis 10:119-127.

Menzies FM, Yneisetti SC, Min K-T (2005) Roles of Drosophila DJ-1 in survival of dopaminergic neurons and oxidative stress. Curr Biol 15:1578-1582.

Meulener M, Whitworth AJ, Armstrong-Gold CE, Rizzu P, Heutink P, Wes PD, Pallanck LJ, Bonini NM (2005) Drosophila DJ-1 mutants are selectively sensitive to environmental toxins associated with Parkinson's disease. Curr Biol 15:1572-1577.

Nakamura K, Bindokas VP, Kowlessur D, Elas M, Milstien S, Marks JD, Halpern HJ, Kang UJ (2001) Tetrahydrobiopterin scavenges superoxide in dopaminergic neurons. J Biol Chem 276:34402-34407.

Neckameyer WS, Weinstein JS (2005) Stress affects dopaminergic signaling pathways in Drosophila. Stress 8:1-15.

Neckameyer WS, White K (1993) Drosophila tyrosine hydroxylase is encoded by the pale locus. J Neurogenet 8:189-199.

Nelson DP, Kiesow LA (1972) Enthalpy of decomposition of hydrogen peroxide by catalase at $25^{\circ} \mathrm{C}$ (with molar extinction coefficient of $\mathrm{H}_{2} \mathrm{O}_{2}$ solution in the u.v.) Anal Biochem 49:474-478.

Park J, Kim SY, Cha GH, Lee SB, Kim S, Chung J (2005) Drosophila DJ-1 mutants show oxidative stress-sensitive locomotive dysfunction. Gene 361:133-139. 
Radyuk SN, Klichko VI, Orr WC (2000) Catalase expression in Drosophila melanogaster is responsive to ecdysone and exhibits both transcriptional and post-transcription regulation. Arch Insect Biochem Physiol 45:79-93.

Rohrdanz E, Schmuck G, Ohler S, Kahl R (2001) The influence of oxidative stress on catalase and MnSOD transcription in astrocytes. Brain Res 900:128-136.

Salvaterra PM, Kitamoto T (2001) Drosophila cholinergic neurons and processes visualized with Gal4/UAS-GFP. Brain Res Gene Expr Patterns $1: 73-82$.

Shimizu K, Matsubara K, Ohtaki K, Shiono H (2003) Paraquat leads to dopaminergic neural vulnerability in organotypic midbrain culture. Neurosci Res 46:523-532.

Shimizu S, Ishii M, Kawakami Y, Momose K, Yamamoto T (1998) Protective effects of tetrahydrobiopterin against nitric oxide-induced endothelial cell death. Life Sci 63:1585-1592.

Stathakis DG, Burton DY, McIvor WE, Krishnakumar S, Wright TRF, O'Donnell JM (1999) The catecholamines up (Catsup) protein of Drosophila melanogaster functions as a negative regulator of tyrosine hydroxylase activity. Genetics 153:361-382.
Stokes AH, Hastings TG, Vrana KE (1999) Cytotoxic and genotoxic potential of dopamine. J Neurosci Res 55:659-665.

Strickland D, Bertoni JM (2003) Parkinson's prevalence estimated by a state registry. Mov Disord 19:318-323.

Sumi-Ichinose C, Urano F, Shimomura A, Sato T, Ikemoto K, Shiraishi H, Senda T, Ichinose H, Nomura T (2005) Genetically rescued tetrahydrobiopterin depleted mice survive with hyperphenylalaninemia and regionspecific monoaminergic abnormalities. J Neurochem 95:703-714.

Tan EK, Khajavi M, Thronby JI, Nagamitsu S, Jankovic J, Ashizawa T (2000) Variability and validity of polymorphism association studies in Parkinson's disease. Neurology 5:533-538.

Uversky VN (2004) Neurotoxicant-induced animal models of Parkinson's disease: understanding the role of rotenone, maneb and paraquat in neurodegeneration. Cell Tissue Res 318:225-241.

Van Den Eeden SK, Tanner CM, Bernstein AL, Fross RD, Leimpeter A, Bloch DA, Nelson LM (2003) Incidence of Parkinson's disease: variation by age, gender, and race/ethnicity. Am J Epidemiol 157:1015-1022.

Warner TT, Schapira AHV (2003) Genetic and environmental factors in the cause of Parkinson's disease. Ann Neurol 53 [Suppl 3]:S16-S25. 